\title{
Esophageal Cancer with Bone Marrow Hyperplasia Mimicking Bone Metastasis: Report of a Case
}

\author{
Hiromi Yasuda Tadanobu Shimura Masato Okigami \\ Shigeyuki Yoshiyama Masaki Ohi Koji Tanaka Yasuhiko Mohri \\ Masato Kusunoki \\ Department of Gastrointestinal and Pediatric Surgery, Mie University Graduate School of \\ Medicine, Tsu, Japan
}

\section{Keywords}

Esophageal cancer · Bone metastasis - Bone marrow hyperplasia · Magnetic resonance imaging

\begin{abstract}
A 63-year-old man visited the clinic with numbness in the right hand. Magnetic resonance imaging demonstrated multiple low-intensity lesions in the cervical vertebrae and sacrum, which was suspicious of cervical bone metastasis. Fluorodeoxyglucose positron emission tomography/computed tomography revealed areas of increased fluorodeoxyglucose uptake in the thoracic esophagus, sternum and sacrum. A flat, elevated esophageal cancer was identified by upper gastrointestinal endoscopy, and the macroscopic appearance indicated earlystage disease. From the cervical, thoracic and abdominal computed tomography images, there were no metastatic lesions except for the bone lesions. To confirm whether the bone lesions were metastatic, we performed bone biopsy. The histopathological diagnosis was bone marrow hyperplasia. It was crucial for treatment planning to establish whether the lesions were distant metastases. Here, we report a case of esophageal cancer with bone marrow hyperplasia mimicking bone metastasis.




\section{Case Reports in Oncology}

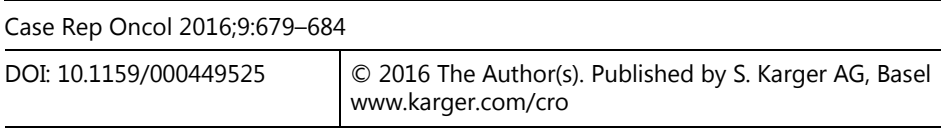

Yasuda et al.: Esophageal Cancer with Bone Marrow Hyperplasia Mimicking Bone Metastasis: Report of a Case

\section{Introduction}

Bone metastases originating from esophageal cancer are uncommon and occur in about $9 \%$ of newly diagnosed patients [1], and prognosis is poor. Generally, treatment for cancer patients with distant metastasis differs from that for patients without distant metastasis. The standard treatment for patients with distant metastasis, such as bone metastasis, is systemic chemotherapy, but the mainstay for patients without distant metastasis is surgical resection. Therefore, diagnosis of metastasis is important for treatment planning, especially when it is difficult to diagnose bone metastasis from magnetic resonance imaging (MRI) and positron emission tomography (PET).

Spinal bone marrow hyperplasia can mimic bone metastasis on MRI and fluorodeoxyglucose (FDG)-PET, which can affect patient management. There were few reports of bone marrow hyperplasia in a patient with cancer [2]. Here, we report a case of esophageal cancer with bone marrow hyperplasia mimicking bone metastasis.

\section{Case Report}

A 63-year-old man presented to his primary care physician with numbness in the right hand. Paralysis of the ulnar nerve was suspected; therefore, he underwent cervical spinal MRI. MRI demonstrated multiple low-intensity areas in his cervical vertebrae, which were suspicious of bone metastases; thus, he was admitted to our hospital. Total spinal MRI was performed and demonstrated several low-intensity lesions in the cervical and thoracic vertebrae, sacrum and sternum (fig. 1a, b, f). On fat-suppressed T2-weighted images, hyperintense areas were not seen in the cervical and thoracic vertebrae (fig. 1d). In contrastenhanced MRI, the low-intensity vertebral lesions lacked contrast (fig. 1c). However, on fat-saturated T1-weighted images, the sacral lesion was not seen because the signal was equivalent to that of the adjacent marrow (fig. 1e). Based on the MRI results, the radiologist reported that the low-intensity areas were equivocal as to whether they represented bone metastases. To investigate the origin of the tumor and spread of metastatic-like lesions, FDGPET/CT was performed. We noted a focal increase in FDG uptake in the intrathoracic esophagus, sternum, and sacrum (fig. 2). The standard uptake values (SUVs) of the thoracic esophagus, sternum, and sacrum were 4.3, 2.8, and 3.0, respectively. As a result of these findings, we performed upper gastrointestinal endoscopy. A flat, elevated esophageal tumor (type 0 -IIa+IIb) was identified at $26-31 \mathrm{~cm}$ from the incisors (fig. 3). The length of the tumor was $\sim 5 \mathrm{~cm}$, lying on the lower third of the thoracic esophagus and extending to the submucosa. The pathological findings of the biopsy specimen revealed squamous cell carcinoma (SCC).

The patient had a history of hypertension, hyperlipidemia, hyperuricemia and chronic anemia. He had no particular family history. He had been smoking 10 cigarettes daily and had consumed $1.7 \mathrm{~L}$ beer daily for 44 years. The laboratory data showed macrocytic anemia (hemoglobin $10.5 \mathrm{~g} / \mathrm{dl}$, mean corpuscular volume $111.5 \mathrm{fl}$ ) and mild liver damage (glutamic oxaloacetic transaminase $53 \mathrm{IU} / \mathrm{l}$; glutamic pyruvic transaminase $8 \mathrm{IU} / \mathrm{l}$ ). The level of serum SCC was within normal limits.

CT of the chest and abdomen did not demonstrate thickening of the esophageal wall, lymph node swelling or distant metastasis. Consequently, we could not exclude bone metastasis from these imaging results. A biopsy of the sternum was performed because bone metastasis from early-stage esophageal cancer is unusual and definitive diagnosis of bone metastasis would exclude the patient from surgery. We selected the sternum for biopsy because 
Yasuda et al.: Esophageal Cancer with Bone Marrow Hyperplasia Mimicking Bone Metastasis: Report of a Case

there were positive findings on both MRI and PET/CT, and it was a lesion upon which we could easily perform a biopsy.

The biopsy revealed that the low-density area in MRI was bone marrow hyperplasia. We diagnosed T1bN0M0 stage I esophageal cancer. So, the patient underwent subtotal esophagectomy with three-field lymph node dissection by thoracoscopy and retrosternal anastomosis of the stomach to the cervical esophagus. Histologically, the tumor was a moderately differentiated SCC at a depth of the submucosal layer, and it had negative surgical margins and positive lymph node metastases [pN2; \#106tbL: left tracheobronchial lymph nodes $(2 / 4)]$. Final diagnosis categorized the tumor as T1bN2M0 and stage II. His postoperative course was uneventful. He has been regularly followed without adjuvant therapy and has remained recurrence-free for 26 months since his operation.

\section{Discussion}

This was a rare case emphasizing the importance of distinguishing between bone metastasis and bone marrow hyperplasia in esophageal cancer. The main treatment for esophageal cancer with distant metastasis, including bone metastasis, is systemic chemotherapy but not surgery. Therefore, exact diagnosis of distant metastasis is important for treatment planning.

Meta-analysis demonstrated that FDG-PET and MRI were significantly more accurate than CT and bone scintigraphy for the diagnosis of bone metastasis [3]. However, it is difficult to distinguish lesions such as compression fracture of the spine due to osteoporosis and bone marrow hyperplasia from bone metastasis, using several imaging methods. There are very few reports of bone marrow hyperplasia with cancer; therefore, we have the potential to misdiagnose bone marrow hyperplasia as bone metastases when not diagnosed carefully.

Bone marrow hyperplasia is a marrow reconversion that refers to repopulating yellow marrow to red marrow. The causes of reconversion are reported to be chronic anemia, treatment with granulocyte-macrophage colony-stimulating factor, and heavy smoking [46]. Our case had chronic anemia and heavy smoking as risk factors for bone marrow hyperplasia.

Typically, bone marrow hyperplasia has similar or lower signal intensity to skeletal muscle on T1- and T2-weighted images and is slightly more hyperintense than muscle on fat-suppressed images [7]. Bone metastatic lesions on T1-weighted images showed lower intensity than the skeletal muscle, and those on T2-weighted images showed increased signal intensity. Therefore, it was difficult to distinguish between bone metastasis and bone marrow hyperplasia. On fat-suppressed T2-weighted images, tumor infiltration typically showed high signal intensity. So, both bone metastasis and bone marrow hyperplasia had similar images on MRI. These results showed lower signal intensity on T1- and T2-weighted images and higher intensity on the fat-suppressed T2-weighted images.

PET/CT are highly sensitive and specific for the detection of bone metastasis [8]. However, bone marrow hyperplasia also showed high uptake compared with adjacent normalappearing vertebrae.

Bordalo-Rodrigues et al. [2] reported a case of a bronchioloalveolar carcinoma with focal nodular bone marrow hyperplasia in dorsal vertebral bodies revealed on FDG-PET and MRI.

After all, like our case, bone biopsy was necessary for their case for a definitive diagnosis. So, bone marrow hyperplasia often shows similar findings to bone metastasis on FDG- 
Yasuda et al.: Esophageal Cancer with Bone Marrow Hyperplasia Mimicking Bone Metastasis: Report of a Case

PET and MRI. Therefore, distinguishing between bone marrow hyperplasia and bone metastasis may be difficult with MRI and PET/CT in some cases.

Shigematsu et al. [9] demonstrated the imaging features of bone marrow hyperplasia and bone metastasis. Their quantitative study showed that mean maximum SUV ( $S U V_{\max }$ ) for bone marrow hyperplasia was significantly lower than that for bone metastasis, and indicated that quantitative assessment using $\mathrm{SUV}_{\max }>3.6$ is useful for differentiating bone marrow hyperplasia from bone metastasis. In our case, on PET/CT, SUV max $_{\text {ax }}$ of the sternum and sacrum was 2.8 and 3.0, respectively, both of which were $<3.6$. In contrast, there was no uptake in the vertebrae. These findings may become one way to distinguish between bone metastasis and bone marrow hyperplasia.

In addition, his study demonstrated that there was no abnormal uptake in areas involved in bone marrow hyperplasia on bone scintigraphs. Therefore, there was a significant difference in the uptake between bone marrow hyperplasia and bone metastasis, and bone scintigraphy can be useful for a differential diagnosis.

In conclusion, it is sometimes hard to distinguish between bone marrow hyperplasia and bone metastasis using various imaging methods in cancer patients. Because esophageal cancer patients may have some risk factors of bone marrow hyperplasia, if physicians encounter a similar patient to the present case, they should take bone biopsy into consideration for accurate diagnosis.

We demonstrate a case of esophageal cancer with bone marrow hyperplasia mimicking bone metastasis.

\section{Statement of Ethics}

All the procedures followed were in accordance with the ethical standards of the responsible committee on human experimentation (institutional and national) and with the Helsinki Declaration of 1964 and later versions. Informed consent was obtained from the patient.

\section{Disclosure Statement}

There are no financial or other relations that could lead to a conflict of interest.

\section{References}

-1 Quint LE, Hepburn LM, Francis IR, Whyte RI, Orringer MB: Incidence and distribution of distant metastases from newly diagnosed esophageal carcinoma. Cancer 1995;76:1120-1125.

-2 Bordalo-Rodrigues M, Galant C, Lonneux M, Clause D, Vande Berg BC: Focal nodular hyperplasia of the hematopoietic marrow simulating vertebral metastasis on FDG positron emission tomography. AJR Am J Roentgenol 2003;180:669-671.

-3 Tachimori Y, Ozawa S, Numasaki H, Fujishiro M, Matsubara H, Oyama T, Shinoda M, Toh Y, Udagawa H, Uno T: Comprehensive registry of esophageal cancer in Japan, 2006. Esophagus 2010;7:7-22.

-4 Vande Berg BC, Lecouvet FE, Galant C, Maldague BE, Malghem J: Normal variants and frequent marrow alterations that simulate bone marrow lesions at MR imaging. Radiol Clin North Am 2005;43:761-770.

-5 Abu-Judeh HH, Naddaf SY, el-Zeftawy HA, Abdel-Dayem HM: G-CSF induced bone marrow hyperplasia: characteristic appearance on total body blood pool and delayed Tc-99m MDP bone scan. Clin Nucl Med 1998;23:39-41.

6 Poulton TB, Murphy WD, Duerk JL, Chapek CC, Feiglin DH: Bone marrow reconversion in adults who are smokers: MR imaging findings. AJR Am J Roentgenol 1993;161:1217-1221. 


\section{Case Reports in Oncology}

\begin{tabular}{l|l}
\hline Case Rep Oncol 2016;9:679-684 \\
\hline DOI: 10.1159/000449525 & $\begin{array}{l}\text { @ 2016 The Author(s). Published by S. Karger AG, Basel } \\
\text { www.karger.com/cro }\end{array}$ \\
\hline
\end{tabular}

Yasuda et al:: Esophageal Cancer with Bone Marrow Hyperplasia Mimicking Bone Metastasis: Report of a Case

$7 \quad$ Siegel MJ: 'MRI of Bone Marrow.' The American Roentgen Ray Society. 2005.

http://www.arrs.org/shopARRS/products/pdf cfm?theFile=s06p sample.pdf.

$>8$ Cheng X, Li Y, Xu Z, Bao L, Li D, Wang J: Comparison of 18F-FDG PET/CT with bone scintigraphy for detection of bone metastasis: a meta-analysis. Acta Radiol 2011;52:779-787.

-9 Shigematsu Y, Hirai T, Kawanaka K, Shiraishi S, Yoshida M, Kitajima M, Uetani H, Azuma M, Iryo Y, Yamashita Y: Distinguishing imaging features between spinal hyperplastic hematopoietic bone marrow and bone metastasis. AJNR Am J Neuroradiol 2014;35:2013-2020.
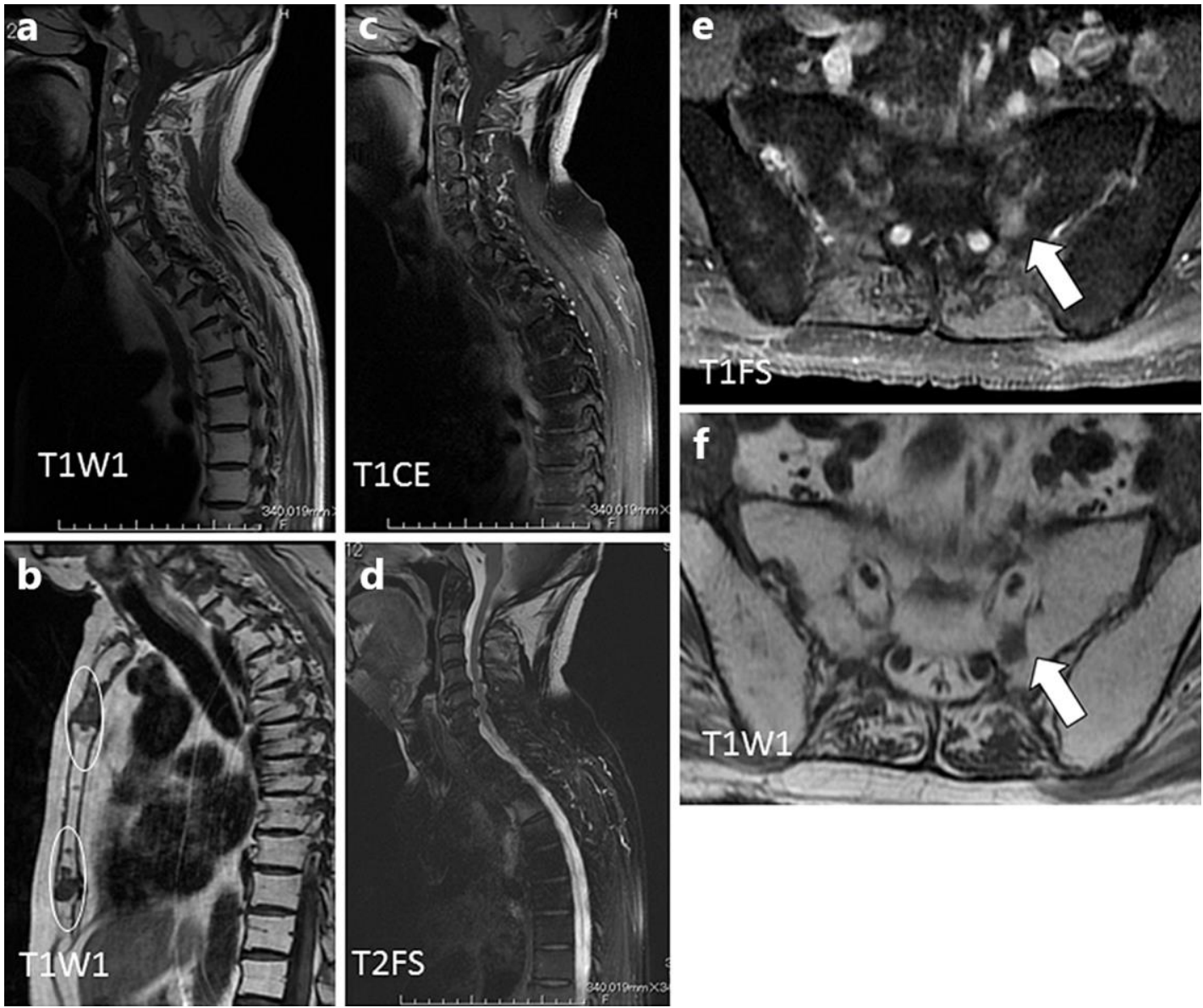

Fig. 1. Sagittal T1-weighted MRI of the cervical and thoracic spine (a) and sternum (white circle) (b) showed several hypointense lesions. Sagittal T1-weighted contrast-enhanced MRI (c) showed that lowintensity lesions of the vertebrae lacked contrast effects. On sagittal fat-saturated T2-weighted images (d), some lesions were not seen because their signal was equivalent to that of adjacent marrow. The sacral lesion, which was detected by positron emission tomography/computed tomography, had a contrast effect (white arrow) on T1-weighted contrast-enhanced MRI (e) but was not seen on fat-saturated T1-weighted images (f). 


\section{Case Reports in Oncology}

\begin{tabular}{l|l}
\hline Case Rep Oncol 2016;9:679-684 \\
\hline DOI: 10.1159/000449525 & $\begin{array}{l}\text { ○ 2016 The Author(s). Published by S. Karger AG, Basel } \\
\text { www.karger.com/cro }\end{array}$ \\
\hline
\end{tabular}

Yasuda et al.: Esophageal Cancer with Bone Marrow Hyperplasia Mimicking Bone Metastasis: Report of a Case
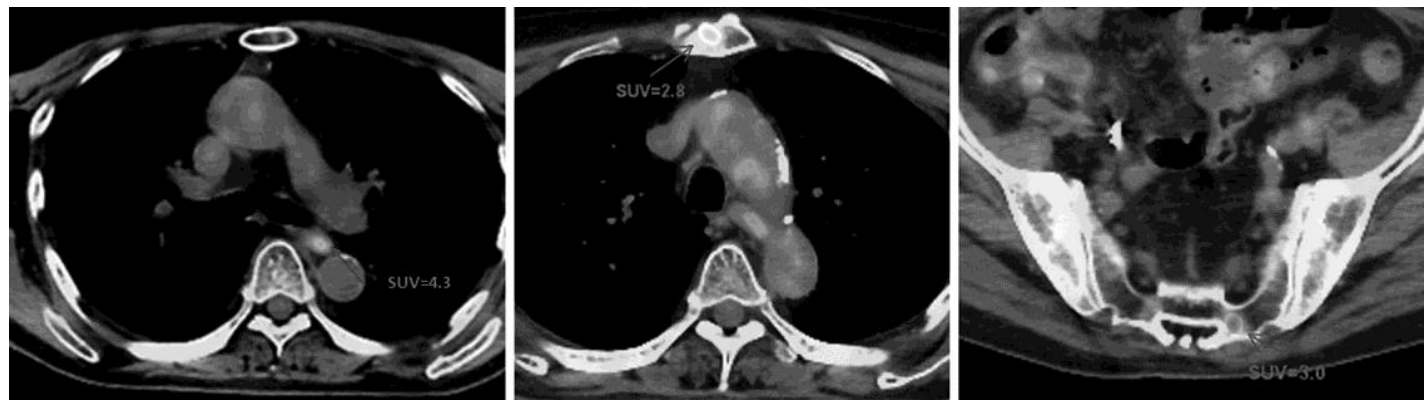

Fig. 2. On FDG-PET/CT for metastasis surveillance, focally increased uptake of FDG was noted in the intrathoracic esophagus (SUV 4.3), sternum (SUV 2.3) and sacrum (SUV 3.0).

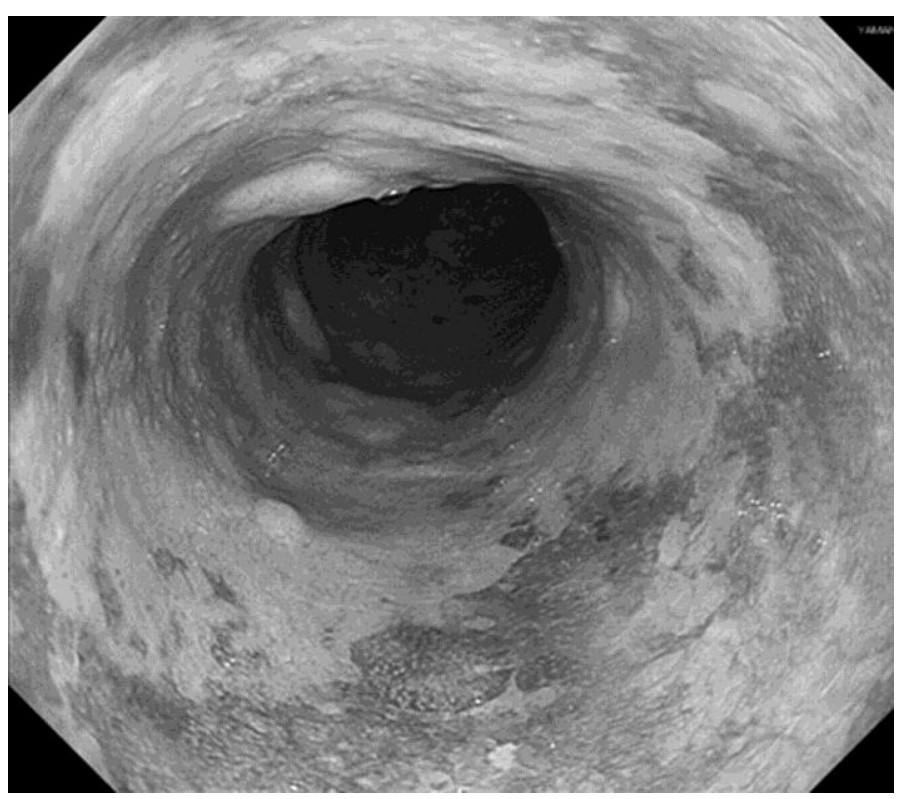

Fig. 3. Esophagoscopy showed type 0-IIa+IIb tumor in the lower intrathoracic esophagus at 26-31 cm from the incisors. After iodine staining, a superficial depressed lesion around the main type 0-IIa tumor was clearly revealed as an iodine-unstained lesion. 